\title{
Potencias de números enteros como suma de impares consecutivos
}

\section{Marco V. Vásquez B.}

Instituto Tecnológico Andrés F. Córdova, Cañar, Ecuador.

Autor para correspondencia: marvas123@hotmail.es

Fecha de recepción: 26 de julio 2013 - Fecha de aceptación: 1 de octubre 2013

\section{RESUMEN}

En este manuscrito, se demuestra que la potencia de $(p+q)$ a cualquier potencia $(n)$, siendo $n$ un número natural puede expresarse como la suma de una serie de impares consecutivos. Variando los índices de los números impares entre un límite inferior que es la mitad de la diferencia entre la potencias $p$ y $q$ del natural $n$ más 1 , y el límite superior, la mitad de esas potencias.

$$
n^{p+q}=\sum_{i=\frac{n^{p}-n^{q}}{2}+1}^{\frac{n^{p}+n^{q}}{2}}(2 i-1)
$$

El algoritmo recalcando su simplicidad, ofrece una oportunidad interesante en las aplicaciones de teorías de números a análisis numérico.

Palabras clave: Número natural, impares, sumatoria, fórmula de potencia.

\section{ABSTRACT}

In this paper it is demonstrated that the power $(p+q)$ of a natural number $(n)$, can be derived as the sum of a series of consecutive odd numbers. The series of the summation is conditioned by a lower and upper boundary, being equal to half the difference plus 1 , and respectively half the sum of the powers $p$ and $q$ of the natural number.

$$
n^{p+q}=\sum_{i=\frac{n^{p}-n^{q}}{2}+1}^{\frac{n^{p}+n^{q}}{2}}(2 i-1)
$$

The algorithm, notwithstanding its simplicity, offers some interesting opportunities in the applications of number theory to numerical analysis.

Keywords: Natural number, odd, sum, power formula.

\section{INTRODUCCIÓN}

Es una certeza, que los números naturales fueron creados por el hombre, en una etapa avanzada de las matemáticas. Estos abren la etapa de la matemática donde la simbología de la cantidad y del valor adquieren un sentido de pragmatismo, de relación directa entre la realidad y los conceptos, lo que dará paso a la operación del conteo, ejercicio netamente de la razón y que responde directamente a un requerimiento del entorno. La suma, por ejemplo, pronostica el resultado de unir dos o más grupos, sin necesidad de tener que contarlos siempre que se conozca los que existen en cada grupo, y así de manera similar, todas las demás, buscando cada vez mayor precisión y complejidad. 
Por lo anterior resulta muy interesante el estudiar las relaciones que existen entre estos números, que a su vez constituyen resultados escondidos, que merecen la pena un espacio de estudio y análisis para entender y generalizarlos, también vale la pena aseverar algo, no hay nada de misterioso ni esotérico en estas relaciones. Son consecuencia de la realidad de su creación, y siempre existirán fundamentos matemáticos que los expliquen.

En este caso, se iniciar indicando que Johann Faulhaber (5 de mayo 1580 - 10 de septiembre 1635), matemático alemán nacido en Ulm, fundador de la escuela de ingenieros en el siglo 17, desarrolló varias fórmulas que permiten calcular la suma de potencias de números naturales consecutivos, cuando el exponente es un impar; que se condensan en el teorema que lleva el nombre del autor, de donde se derivan en fórmulas que sirven especialmente en las matemáticas computacionales. Bazsó y col. (2012) publicaron un refinamiento de ese teorema que generaliza para otros exponentes. Muchos trabajos se han presentado en esta línea, buscando siempre construir una fórmula que sintetice la suma de potencias de igual exponente. Sin detenerse a entender y explicar que dada una de esas potencias pueden expresarse como la sumatoria de números impares consecutivos, si bien en el año 2007 la página WEB http://gaussianos.com/ publicó unos resultados que muestran como la suma de impares consecutivos da cómo resultado un número cuadrado perfecto sin detenerse a explicar la razón de este resultado, peor aún, de generalizarlo a exponentes enteros distintos de 2.

Este documento se limita a trabajar en los naturales impares. Básicamente se intenta utilizarlos para construir potencias de números enteros, generando por su puesto igualdades que merecen ser entendidas. Los algoritmos computacionales buscan siempre generar números con cálculos lo más simples posible. Estos resultados permiten construir cualquier potencia en base de sumar algunos números impares consecutivos, por lo que la utilidad de estos resultados en matemáticas computacionales (Sen y Agarwal, 2006) es segura, ayudando a ello la cualidad de los números impares que en la notación binomial todos terminan con la unidad, lo que obviamente facilita su representación práctica y las operaciones. Consecuentemente, se pretende ser lo más explícitos en cada desarrollo, sin el afán de irrespetar la formalidad que exige las matemáticas, mas bien siempre buscando que los procesos se sujeten a la realidad concreta que fundamenta esta ciencia y que deben estar presentes siempre. Por lo tanto, se busca proponer procesos simples, de fácil comprensión, con simbología clara para mostrar lo amigable de la madre de las ciencias, se recurrirá además a las equivalencias que existen entre los procesos numéricos, algébricos y geométricos para mostrar que los mismos son escenarios distintos de una misma realidad, por lo que obligatoriamente deben reflejar exactamente lo mismo.

Este trabajo muestra una característica que se da exclusivamente en el conjunto de los números impares, esto es que sumando elementos consecutivos se pueda obtener el resultado de cualquier potencia, resultado de gran ayuda para las matemáticas computacionales donde se requiere que las operaciones sean lo más simples posible, y de hecho el algoritmo de sumar impares consecutivos es de fácil programación, y más simple en su desarrollo operativo, es entonces en estos dos conceptos donde radica la relevancia de este manuscrito.

\section{CARACTERIZACIÓN DE LOS NÚMEROS IMPARES}

El conjunto de los números impares positivos es el siguiente:

$$
\{1,3,5,7,9,11,13,15,17,19,21,23,25,27,29, \ldots\}
$$

que cumplen la condición de no ser múltiplos de dos, y además es ordenado, iniciando en 1 y extendiéndose hacia el infinito, por lo que es un conjunto de cardinalidad infinita. Los números impares está relacionados con el conjunto de los naturales según la relación siguiente:

$$
a_{n}=2 n-1
$$

donde $n$ es un número natural cualquiera, distinto de cero.

Si extendemos el conjunto donde se define $\mathrm{n}$ y aceptamos al cero y a los números negativos, ampliaremos este conjunto base a todos los números enteros incluido el cero y con la misma relación ya indicada construiremos el conjunto de todos los números impares existentes. Es de aclarar que los 
números impares pueden ser positivos o negativos. Además se debe mencionar que existe otra relación que también puede caracterizar los números impares y es la siguiente:

$$
a_{n}=2 n+1
$$

para cualquier $n$ entero cero.

\section{EL CUADRADO DE UN NÚMERO}

En la Fig. 1 se observa que el cuadrado de un número natural es igual al cuadrado del natural que lo procede más dos veces ese mismo número y más uno, esto intentemos demostrar para generalizar.

ENUNCIADO 1: El cuadrado de un número natural n, es igual al cuadrado del natural inmediatamente menor $n-1$, más un número impar que resulta de multiplicar por 2 el número (n1) y sumar 1 .

$$
P D^{l}: n^{2}=(n-1)^{2}+(2(n-1)+1)
$$

DEMOSTRACIÓN: Simplemente desarrollamos $(\mathrm{n}-1)^{2}$ y tenemos:

$$
(n-1)^{2}+(2(n-1)+1)=n^{2}-2 n+1+2 n-2+1=n^{2}
$$

Lo que queríamos demostrar (LQQD).

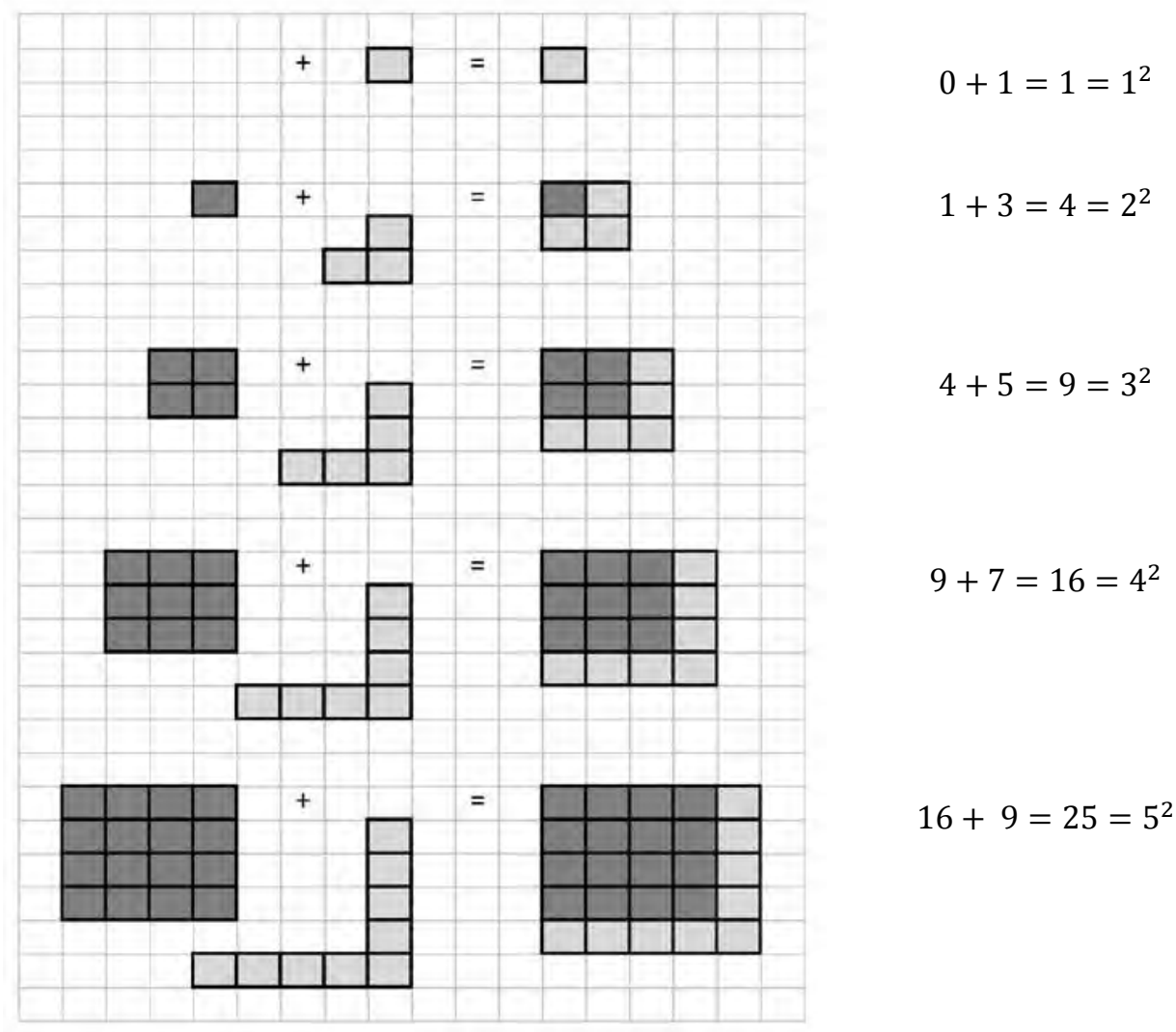

Figura 1. Presentación gráfica del cuadrado de los números naturales.

\footnotetext{
${ }^{1}$ PD: Por demostrar
} 
Es posible concluir entonces que un cuadrado es igual a una sumatoria sucesiva de números impares (De Santa Cruz, 1794; De Knudt, 1993), como se observa en los siguientes ejemplos:

$$
\begin{aligned}
& 1^{2}=1 \\
& 2^{2}=1^{2}+3=1+3=4 \\
& 3^{2}=2^{2}+5=1^{2}+3+5=1+3+5=9 \\
& 4^{2}=3^{2}+7=2^{2}+5+7=1^{2}+3+5+7=1+3+5+7=16 \\
& 5^{2}=4^{2}+9=3^{2}+7+9=2^{2}+5+7+9=1^{2}+3+5+7+9=1+3+5+7+9=25 \\
& 6^{2}=\ldots=1+3+5+7+9+11=36 \\
& 7^{2}=\ldots=1+3+5+7+9+11+13=49 \\
& 8^{2}=\ldots=1+3+5+7+9+11+13+15=64 \\
& 9^{2}=\ldots=1+3+5+7+9+11+13+15+17=81 \\
& 10^{2}=\ldots=1+3+5+7+9+11+13+15+17+19=100
\end{aligned}
$$

y así sucesivamente.

ENUNCIADO 2: El cuadrado de un número natural positivo $\boldsymbol{n}$ igual a la suma de los impares sucesivos, iniciando en 1 y culminando en $2 \boldsymbol{n}-1$ (Ec. 1).

$P D$ :

$$
\sum_{i=1}^{n}(2 i-1)=n^{2}
$$

DEMOSTRACIÓN: Debemos recordar simplemente que:

y tendremos que:

$$
\sum_{i=1}^{n} i=\frac{n(n+1)}{2}
$$

$$
\begin{gathered}
\sum_{i=1}^{n}(2 i-1)=2 \sum_{i=1}^{n} i-\sum_{i=1}^{n} 1=2 \frac{n(n+1)}{2}-n \\
\sum_{i=1}^{n}(2 i-1)=n(n+1)-n=n^{2}+n-n=n^{2} \quad(L Q Q D)^{2}
\end{gathered}
$$

Con lo que queda demostrado que el cuadrado de cualquier número natural, $\boldsymbol{n}$, puede expresarse como una sumatoria de números impares sucesivos desde 1 hasta $(2 \boldsymbol{n}-1)$.

\section{EL CUBO DE UN NÚMERO ENTERO}

La idea intuitiva que permite este resultado es que si se multiplica el cuadrado de un número entero por ese mismo número, el resultado es el cubo, lo que se puede expresar también indicando que si se suma el cuadrado de un número entero, tantas veces como la cantidad que representa ese entero, el resultado equivaldrá también al cubo buscado.

Es decir:

$$
\boldsymbol{n}^{3}=\boldsymbol{n}^{2} * n=\begin{gathered}
n^{2}+n^{2}+\cdots \ldots \ldots+n^{2} \\
<-----------> \\
n \text { veces }
\end{gathered}
$$

\footnotetext{
${ }^{2}$ LQQD: Lo que queríamos demostrar
} 
Más la suma indicada puede presentarse, en el caso de que $n$ sea impar de la siguiente forma:

$$
\begin{aligned}
n^{3}= & \left(n^{2}-n\right)+\left(n^{2}-n+2\right)+\left(n^{2}-n+4\right)+\cdots+\left(n^{2}-2\right)+\left(n^{2}\right) \\
& +\left(n^{2}+2\right)+\cdots+\left(n^{2}+n-4\right)+\left(n^{2}+n-2\right)+\left(n^{2}+n\right)
\end{aligned}
$$

Donde también se tienen $n$ términos, todos impares consecutivos de tal forma que al sumar se compensan entre sí, basados en lo siguiente:

$$
\begin{gathered}
\left(n^{2}+n\right)+\left(n^{2}-n\right)=2 n^{2} \\
\left(n^{2}+n-2\right)+\left(n^{2}-n+2\right)=2 n^{2}
\end{gathered}
$$

Debiendo por tanto tomar $(n-1) / 2$, elementos a la izquierda de $n^{2}$ e igual cantidad a la derecha.

EJEMPLO: si deseamos obtener $7^{3}$, tomaremos como base $7^{2}$, y a la izquierda ubicaremos los 3 , en razón de que $((7-1) / 2=3)$ impares consecutivos, estos serían $(43,45$ y 47$)$, y así mismo a la derecha tomaremos los tres impares consecutivos (51, 53 y 55), y tendremos que: $43+45+47+$ $49+51+53+55=343=7^{3}$.

En el caso de que $n$ sea par, $n^{2}$ también lo será, los dos impares medios consecutivos serán $\left(n^{2}-1\right)$ y $\left(n^{2}+1\right)$, y además tomaremos a la izquierda y a la derecha $\left(\frac{n}{2}-1\right)$ elementos con los que tendríamos los $n$ elementos que dan el resultado deseado.

EJEMPLO: Supongamos se desea obtener $8^{3}$, los dos medios serían $\left(8^{2}-1\right)=63$ y $\left(8^{2}+1\right)=65$, y a cada lado tendríamos que obtener 3 números impares consecutivos $(8 / 2-1)=3$, que serían $(57$, 59 y 61$)$ para la izquierda y $(67,69$ y 71$)$ para la derecha, y se tendría: $\mathbf{5 7}+\mathbf{5 9}+\mathbf{6 1}+\mathbf{6 3}+\mathbf{6 5}+$ $67+69+71=512=8^{3}$.

Pero adicionalmente, otra forma de obtener como resultado $n^{3}$ es sumar $n^{2}$ veces el número $n$, es decir:

$$
n^{3}=n * n^{2}=\begin{gathered}
n+n+n+\cdots+n+n+n \\
<--------->> \\
n^{2} \text { veces }
\end{gathered}
$$

Que buscando números impares que se compensen, con $n$ impar, sería:

$$
\begin{gathered}
\left(n-n^{2}+1\right)+\left(n-n^{2}+3\right)+\left(n-n^{2}+5\right)+\cdots(n-2)+n+(n+2)+\cdots+\left(n+n^{2}-5\right) \\
+\left(n+n^{2}-3\right)+\left(n+n^{2}-1\right)
\end{gathered}
$$

Tenemos $n^{2}$ términos cuya sumatoria es $n^{3}$

$$
\begin{aligned}
& \text { EJEMPLO: } 7^{3}=\left(7-7^{2}+1\right)+\left(7-7^{2}+3\right)+\left(7-7^{2}+5\right)+\ldots+(7-2)+7+(7+2)+\ldots+\left(7+7^{2}-\right. \\
& 5)+\left(7+7^{2}-3\right)+\left(7+7^{2}-1\right)=-41-39-37-35-33-31-29-27-25-23-21-19-17- \\
& 15-13-11-9-7-5-3-1+1+3+5+7+9+11+13+15+17+19+21+23+25+27 \\
& +29+31+33+35+37+39+41+43+45+47+49+51+53+55 .
\end{aligned}
$$

Que son 49 términos $\left(7^{2}\right)$, cuya suma, en vista de que existen valores negativos y positivos se reduce a:

$7^{3}=43+45+47+49+51+53+55=343$ (expresión idéntica a lo visto en el ejemplo anterior)

Si $n$ es par, la sumatoria es:

$$
\begin{aligned}
\left(n-n^{2}+1\right)+\left(n-n^{2}+3\right)+ & \left(n-n^{2}+5\right)+\cdots+(n-1)+(n+1)+\cdots+\left(n+n^{2}-5\right) \\
& +\left(n+n^{2}-3\right)+\left(n+n^{2}-1\right)
\end{aligned}
$$

Donde también se tiene $n^{2}$ términos.

$$
\begin{aligned}
& \text { EJEMPLO: } 8^{3}=\left(8-8^{2}+1\right)+\left(8-8^{2}+3\right)+\left(8-8^{2}+5\right)+\ldots+7+9+\ldots+\left(8+8^{2}-5\right)+\left(8+8^{2}-\right. \\
& 3)+\left(8+8^{2}-1\right)=-55-53-51-49-47-45-43-41-39-37-35-33-31-29-27-25-23- \\
& 21-19-17-15-13-11-9-7-5-3-1+1+3+5+7+9+11+13+15+17+19+21+23 \\
& +25+27+29+31+33+35+37+39+41+43+45+47+49+51+53+55+57+59+61 \\
& +63+65+67+69+71
\end{aligned}
$$

Que así mismo son 64 términos $\left(8^{2}\right)$, y que anulando positivos con negativos se reduce a: 
$8^{3}=57+59+61+63+65+67+69+71=512$, repitiendo también el resultado de un ejemplo anterior.

\section{LA POTENCIA CUARTA DE UN ENTERO, EXPRESADA COMO SUMATORIA DE IMPARES CONSECUTIVOS}

Como es conocido, si a un número al cuadrado, le elevamos al cuadrado, el resultado será el número inicial elevado al cuadrado, o que en fórmula sería: $\left(n^{2}\right)^{2}=n^{4}$

$\mathrm{Y}$ como se tiene que:

$$
\sum_{i=1}^{n}(2 i-1)=n^{2}
$$

Se obtiene la Ec. 2:

$$
n^{4}=\left(n^{2}\right)^{2}=\sum_{i=1}^{n^{2}}(2 i-1)
$$

DEMOSTRACIÓN: Como $n$ es entero positivo, consecuentemente $\mathrm{n}^{2}$ también lo será y se tendrá que:

$$
\sum_{i=1}^{n^{2}}(2 i-1)=2 \sum_{i=1}^{n^{2}} i-\sum_{i=1}^{n^{2}} 1
$$

Que utilizando las fórmulas de sumatorias de potencias consecutivas, se tendrá que:

$$
\begin{gathered}
\sum_{i=1}^{n^{2}}(2 i-1)=2 \frac{n^{2}\left(n^{2}+1\right)}{2}-n^{2} \\
\sum_{i=1}^{n^{2}}(2 i-1)=n^{2}\left(n^{2}+1\right)-n^{2}=n^{4}+n^{2}-n^{2}=\boldsymbol{n}^{4} \quad \text { (LQQD) }
\end{gathered}
$$

EJEMPLO: Con $n=5$.

$$
5^{4}=\sum_{i=1}^{5^{2}}(2 i-1)=\sum_{i=1}^{25}(2 i-1)
$$

$5^{4}=1+3+5+7+9+11+13+15+17+19+21+23+25+27+29+31+33+35+37+$ $39+41+43+45+47+49=625$

El resultado de la Ec. (2), puede generalizarse, de la siguiente forma (Ec. 3):

$$
n^{2 p}=\left(n^{p}\right)^{2}=\sum_{i=1}^{n^{p}}(2 i-1)
$$

con $n$ y $p$ enteros positivos.

DEMOSTRACIÓN: Esta demostración es muy similar a la anterior. Como $n$ es entero positivo, consecuentemente $n^{p}$ también lo será y se tendrá que: 


$$
\sum_{i=1}^{n^{p}}(2 i-1)=2 \sum_{i=1}^{n^{p}} i-\sum_{i=1}^{n^{p}} 1
$$

Que utilizando las fórmulas de sumatorias de potencias consecutivas, se tendrá que:

$$
\begin{gathered}
\sum_{i=1}^{n^{p}}(2 i-1)=2 \frac{n^{p}\left(n^{p}+1\right)}{2}-n^{p} \\
\sum_{i=1}^{n^{p}}(2 i-1)=n^{p}\left(n^{p}+1\right)-n^{p}=n^{2 p}+n^{p}-n^{p}=\boldsymbol{n}^{2 p} \text { (LQQD) }
\end{gathered}
$$

Mas existen algunas otras combinaciones que permiten también trabajar las potencias de $n^{4}$.

$$
\begin{gathered}
n+n+n+\cdots+n+n+n \\
n^{4}=n * n^{3}=<----------> \\
n^{3} \text { veces } \\
n^{4}=n^{2} * n^{2}=<-------------> \\
n^{2} \text { veces } \\
n^{3}=n^{3}+n=n^{3}+\cdots+n^{3}+n^{3}+n^{3} \\
n \text { veces }
\end{gathered}
$$

Que podrían trabajarse para obtener de tres maneras el resultado deseado.

Generando la siguiente fórmula:

Si $n, p$ y $q$ son enteros positivos, se obtiene la Ec. 4 :

$$
n^{p}=\sum_{i=1}^{n^{q}}\left(n^{(p-q)}-n^{q}+2 i-1\right)
$$

Es de indicar que ya sea $n$ para o impar, la expresión $\left(n^{(p-q)}-n^{q}\right)$, siempre dará como resultado un numero par que además es invariante con respecto a $i$, en cambio la expresión $2 i-1$, caracteriza los números impares, y va variando justamente conforme varia el valor de $i$, con lo cual se tiene que la sumatoria propuesta es justamente de números impares consecutivos.

DEMOSTRACIÓN: Como $n, p$ y $q$ son enteros positivos, $n^{p}$ y $n^{q}$ son enteros positivos, por lo tanto.

$$
\sum_{i=1}^{n^{q}}\left(n^{(p-q)}-n^{q}+2 i-1\right)=\left(n^{(p-q)}-n^{q}-1\right)\left(n^{q}\right)+2 \sum_{i=1}^{n^{q}}(i)
$$

Ya que $\left(n^{(p-q)}-n^{q}-1\right)$ es invariante con respecto a $i$, y se generan $n^{q}$ términos, y además con la fórmula de sumatoria de naturales consecutivos, se tiene que:

$$
\begin{gathered}
\sum_{i=1}^{n^{q}}\left(n^{(p-q)}-n^{q}+2 i-1\right)=\left(n^{(p-q)}-n^{q}-1\right)\left(n^{q}\right)+2 \frac{n^{q}\left(n^{q}+1\right)}{2} \\
=n^{p}-n^{2 q}-n^{q}+n^{2 q}+n^{q}=n^{p} \quad(L Q Q D)
\end{gathered}
$$

EJEMPLO: $n=9, p=5, q=1$ : 
se tienen 9 términos:

$$
9^{5}=\sum_{i=1}^{9}\left(9^{4}-9^{1}+2 i-1\right)=\sum_{i=1}^{9}(6561-9+2 i-1)
$$

$$
9^{5}=6553+6555+6557+6559+6561+6563+6565+6567+6569=\mathbf{5 9 0 4 9}
$$

En caso de que $n=9, p=5, q=2$ :

se tienen 81 términos:

$$
9^{5}=\sum_{i=1}^{9^{2}}\left(9^{3}-9^{2}+2 i-1\right)=\sum_{i=1}^{81}(729-81+2 i-1)
$$

$$
\begin{gathered}
9^{5}=649+651+653+655+657+\cdots+727+729+781+\cdots+801+803+805 \\
+807+809=\mathbf{5 9 0 4 9}
\end{gathered}
$$

Ahora, $\operatorname{si} n=9, p=5, q=3$ veamos:

$$
9^{5}=\sum_{i=1}^{9^{3}}\left(9^{2}-9^{3}+2 i-1\right)=\sum_{i=1}^{729}(81-729+2 i-1)
$$

se tienen 729 términos:

$$
\begin{gathered}
9^{5}=-647-645-643-\cdots-5-3-1+1+3+5+\cdots+643+645+647+649+ \\
651+653+655+657+\cdots+727+729+781+\cdots+801+803+805+807+809= \\
\mathbf{5 9 0 4 9}
\end{gathered}
$$

Muchos de estos términos se anulan entre positivos y negativos, quedando una sumatoria de los impares consecutivos desde 649 hasta 809 , que ya obtuvimos en otro caso.

Por último, veamos que pasa para el caso $n=9, p=5, q=4$ :

$$
9^{5}=\sum_{i=1}^{9^{4}}\left(9^{1}-9^{4}+2 i-1\right)=\sum_{i=1}^{6561}(9-6561+2 i-1)
$$

se tiene 6561 términos:

$$
\begin{gathered}
9^{5}=-6551-6549-6547-\cdots-5-3-1+1+3+5+\cdots+6547+6549+6551+ \\
6553+6555+6557+6559+6561+6563+6565+6567+6569=\mathbf{5 9 0 4 9}
\end{gathered}
$$

De igual forma se anulan una gran cantidad de números positivos y negativos, y se repite el resultado ya conocido.

\section{GENERALIZACIÓN}

El resultado que se obtiene de la Ec. 1, permite obtener un resultado que generaliza estas igualdades:

Sabemos que, para cualquier $n$ entero positivo, se tiene que:

$$
\sum_{i=1}^{n}(2 i-1)=n^{2}
$$

Si reemplazamos $n$ por $n^{q}$, donde $q$ es un entero positivo cualquiera, se tendrá: 


$$
\sum_{i=1}^{n^{q}}(2 i-1)=\left(n^{q}\right)^{2}=n^{2 q}
$$

Y así mismo con $n^{p}$, donde $p$ es un entero positivo cualquiera, se tendrá:

$$
\sum_{i=1}^{n^{p}}(2 i-1)=\left(n^{p}\right)^{2}=n^{2 p}
$$

Además, si construimos el valor $\frac{n^{p}+n^{q}}{2}$, sabemos que para $n$ par o impar, este resultado será un número entero positivo, y el valor $\frac{n^{p}-n^{q}}{2}$, es otro número entero.

Por tanto, podemos construir las sumatoria de series de números impares consecutivos:

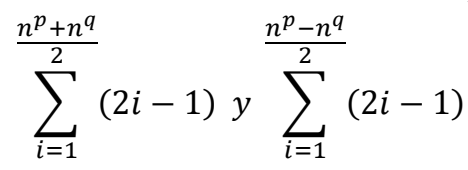

Pudiendo así construir una serie cuyo primer índice no sea 1 sino el menor de los números construidos más uno $\left(\frac{n^{p}-n^{q}}{2}+1\right)$ y que concluya en el mayor de los números construidos $\left(\frac{n^{p}+n^{q}}{2}\right)$, cuya representación es la siguiente operación:

$$
\begin{gathered}
n^{p}-n^{q}+n^{p}-n^{q}+1+n^{p}-n^{q}+2+n^{p}-n^{q}+3+\cdots+n^{p}+n^{q}-3+n^{p} \\
+n^{q}-2+n^{p}+n^{q}-1
\end{gathered}
$$

Cuyo resultado expondremos a continuación: Para n, p, q, números enteros positivos, la suma de los impares consecutivos construidos con la forma $(2 i-1)$, con y desde $\left(\frac{n^{p}-n^{q}}{2}+1\right)$ hasta $\left(\frac{n^{p}+n^{q}}{2}\right)$ es igual a $n^{p+q}$.

Dicho de otra manera, se cumple la siguiente igualdad (Ec. 5):

$$
n^{p+q}=\sum_{i=\frac{n^{p}-n^{q}}{2}+1}^{\frac{n^{p}+n^{q}}{2}}(2 i-1)
$$

Comentarios críticos sobre la ecuación 5:

- $\quad$ Si $n=0$ o negativo, la ecuación no tiene sentido.

- $\quad$ Si $p$ es cero, o negativo, la ecuación se cumple.

- $\quad$ Si $q$ es cero, la sumatoria es de un único término y se cumple.

- $\quad \operatorname{Si} q$ es menor que cero la sumatoria no tiene sentido.

DEMOSTRACIÓN: Es sabido que:

$$
\sum_{i=\frac{n^{p}-n^{q}}{2}+1}^{\frac{n^{p}+n^{q}}{2}}(2 i-1)=\sum_{i=1}^{\frac{n^{p}+n^{q}}{2}}(2 i-1)-\sum_{i=1}^{\frac{n^{p}-n^{q}}{2}}(2 i-1)
$$

Por la Ec. 1 se sabe que:

$$
\sum_{i=1}^{\frac{n^{p}+n^{q}}{2}}(2 i-1)=\left(\frac{n^{p}+n^{q}}{2}\right)^{2}
$$

Y además: 
Por lo tanto:

$$
\sum_{i=1}^{\frac{n^{p}-n^{q}}{2}}(2 i-1)=\left(\frac{n^{p}-n^{q}}{2}\right)^{2}
$$

$$
\sum_{i=\frac{n^{p}-n^{q}}{2}+1}^{\frac{n^{p}+n^{q}}{2}}(2 i-1)=\left(\frac{n^{p}+n^{q}}{2}\right)^{2}-\left(\frac{n^{p}-n^{q}}{2}\right)^{2}
$$

Que es una diferencia de cuadrados que es igual a la suma por la diferencia de sus bases.

$$
\begin{gathered}
\sum_{i=\frac{n^{p}-n^{q}}{2}+1}^{\frac{n^{p}+n^{q}}{2}}(2 i-1)=\left(\left(\frac{n^{p}+n^{q}}{2}\right)+\left(\frac{n^{p}-n^{q}}{2}\right)\right)\left(\left(\frac{n^{p}+n^{q}}{2}\right)-\left(\frac{n^{p}-n^{q}}{2}\right)\right) \\
=\left(\frac{2 n^{p}}{2}\right)\left(\frac{2 n^{p}}{2}\right)=n^{p} \cdot n^{q}=\boldsymbol{n}^{(\boldsymbol{p}+\boldsymbol{q})} \quad(L Q Q D)
\end{gathered}
$$

EJEMPLO: Utilizando el último teorema demostrado calcular, la sumatoria de impares consecutivos que den como resultado $7^{9}$.

Para aplicar la fórmula (5), definiremos $n=7, p=5$ y $q=4$ :

Y tendremos lo siguiente:

$$
n^{p+q}=\sum_{i=\frac{n^{p}-n^{q}}{2}+1}^{\frac{n^{p}+n^{q}}{2}}(2 i-1)
$$

Es sabido que $7^{4}=2401$ y $7^{5}=16807$, por tanto:

$$
7^{9}=\sum_{i=\frac{7^{5}-7^{4}}{2}+1}^{\frac{7^{5}+7^{4}}{2}}(2 i-1)
$$

$$
\begin{gathered}
\frac{7^{5}+7^{4}}{2}=\frac{16807+2401}{2}=\frac{19208}{2}=9604 \\
\frac{7^{5}-7^{4}}{2}+1=\frac{16807-2401}{2}+1=\frac{14406}{2}+1=7204 \\
7^{9}=\sum_{i=7204}^{9602}(2 i-1)= \\
+14407+14409+14411+14413+\cdots+19195+19197+19199
\end{gathered}
$$

Resultado correcto que resulta de sumar números impares consecutivos y que obviamente da un resultado correcto, se puede comprobar que: $7^{9}=\mathbf{4 0 3 5 3 6 0 7}$

\section{CONCLUSIONES}

Los resultados que aquí se han obtenido, sobre todo muestran como cualquier potencia de un número entero puede obtenerse como la sumatoria de varios números impares consecutivos. Está claro también que el cálculo en sí no se simplifica. Muchas veces se requiere una cantidad grande de impares consecutivos para sumarlos y obtener el resultado deseado. Mas el poder entender esta propiedad de 
los impares resulta de enorme importancia en teoría de números y quizá ayude en algoritmos numéricos. Estos resultados cobran especial interés en matemáticas computacionales, donde es segura su aplicabilidad, ya que como se mencionado permiten descomponer a cualquier potencia como la sumatoria de números impares consecutivos, simplemente números a la primera potencia. Además, las relaciones de por sí representan interesantes equivalencias en la teoría de números. Lo primordial es que permite entender fundamentadamente resultados de los números, que se dan exclusivamente en base de la naturaleza de éstos.

\section{BIBLIOGRAFÍA}

Bazsó, A., H.M. Pintér, C. Srivastava, 2012. A refinement of Faulhaber's theorem concerning sums of powers of natural numbers. Appl. Math. Lett., 25, 486-489.

De Santa Cruz, M.G., 1794. Dorado contador. Aritmética especulativa y práctica. Imprenta de don Benito Cano, Madrid, España, pp. 4-6.

Gaussianos.com, 2007. Sumando números impares. Descargado de http://gaussianos.com en 2011.

Knuth, D.E., 1993. Johann Faulhaber and sum of powers. Math. Comp., 61(203), 277-294.

Sen, S.K., H. Agarwal, 2006. 2n in scientific computation and beyond. Math. Comput. Modelling, 43, 658-672. 